\title{
Forum
}

\section{À propos de la charte concernant la modélisation comme outil d'accompagnement}

\author{
Francis Laloë \\ Statisticien halieute, IRD, UMR C3ED, BP 64501, 34394 Montpellier cedex 5, France
}

La réflexion sur la modélisation comme outil d'accompagnement proposée par le collectif ComMod, dans la revue Natures Sciences Sociétés (2005, 13, 2, 165-168) est relative à un sujet passionnant pour des raisons à la fois pratiques et théoriques. Les questions déontologiques soulevées par le recours à la modélisation dans les recherches interdisciplinaires finalisées sont essentielles et la réflexion proposée est de ce point de vue très utile. Comme statisticien impliqué dans des programmes de recherche présentant nombre des caractéristiques de ceux qui sont à la base de cette réflexion - et donc comme acteur de «modélisation d'accompagnement »-, je me suis évidemment senti très concerné par cette démarche. Et ce, d'autant plus que je partage très largement le souci d'accorder une place centrale aux «questions de terrain ", qui est au cœur de la démarche proposée à la réflexion.

Cependant, l'ensemble du texte m'a laissé perplexe, car certains des choix sur lesquels il repose me posent question. C'est tout d'abord celui de sa forme : pourquoi se doter d'une «doctrine », aussi « réfutable » soit-elle, et pourquoi la présenter dans le cadre d'une «charte»? C'est ensuite le statut central - et apparemment exclusif donné à la modélisation pour caractériser la posture d'un groupe multidisciplinaire. C'est également la sélection, au sein de la modélisation, de démarches et d'outils particuliers. C'est enfin la définition a priori de limites aux questions auxquelles il est envisagé de pouvoir répondre.

La posture décrite en introduction s'appuie sur l'idée que les recherches «impliquées » associent des questions théoriques et des questions « de terrain » bien distinctes les unes des autres. Cela conduit à afficher un certain nombre d'objectifs avec lesquels on ne peut guère, en première analyse, être en désaccord. Il est en effet souhaitable de considérer que « toutes les idées à la base de la modélisation ont comme vocation d'être remises en cause

Auteur correspondant : laloe@mpl.ird.fr au contact du terrain ». Il l'est certes aussi de «n'avoir aucune hypothèse implicite dans l'expérimentation [... ce qui] implique le développement de procédures de mise en évidence de telles hypothèses ». Il l'est encore de s'intéresser aux « processus de validation de cette démarche de recherche, sachant qu'il n'existe pas de théorie générale de la validation des modèles et que des procédures autres que celles utilisées dans les modèles bio-physicomathématiques sont à envisager ».

Tout cela est à la fois vrai, banal et complexe, au point qu'il me semble très difficile de distinguer ce qui est réfutable de ce qui ne l'est pas. Ainsi, si des procédures de mise en évidence des hypothèses implicites sont à envisager, se pose le problème de leur validation. En effet, si la découverte d'une hypothèse de cette nature peut apparaître comme une démonstration de leur efficacité, l'absence d'une telle découverte devient une indication de satisfaction de l'hypothèse d'absence de telles hypothèses...

En fait, je crois bien qu'une hypothèse implicite est faite dans la charte qui nous est proposée, en ne considérant, au sein de l'ensemble très large de méthodes, outils, démarches, concepts pouvant être réunis sous le terme de modélisation, qu'un sous-ensemble organisé autour des systèmes multi-agents (SMA). L'existence d'autres voies et outils de modélisation n'est évoquée que de façon sommaire, en les réunissant sous le qualificatif de «bio-physico-mathématique ». Cela ne me semble pas acceptable, sauf à considérer que cela ne mérite pas d'explicitation. Je ne suis pas du tout sûr qu'existe une théorie particulière de validation valant pour l'ensemble des éléments de cet amalgame.

Plus problématiques sont les limites que donnent les auteurs de la charte aux questions pour lesquelles elle est censée avoir validité. Il est ainsi dit que la «modélisation d'accompagnement conduit les acteurs à partager 
des représentations et des simulations comprenant les actions possibles (règles, aménagement...) qu'ils envisagent sur le milieu ». Cet objectif affiché est justifié, mais la modélisation proposée permet-elle de formaliser - et donc de comprendre - les actions possibles des acteurs ou bien des actions possibles d'acteurs? Et ces actions sont-elles toutes compréhensibles par la méthode proposée? La réponse à ces questions est, d'une certaine façon, donnée dans la phrase suivante indiquant que la modélisation d'accompagnement «ne prend pas en charge les autres étapes possibles du processus, qui concerneraient une expertise plus quantifiée (taille d'un aménagement, production estimée, etc.) ». Est-il certain que ces estimations ne constituent pas des « questions de terrain » possibles? Que doit-on dire à des acteurs qui les poseraient explicitement et attendraient (ou diraient attendre) une réponse avant de décider?

Ces remarques critiques ne concernent pas la qualité de la démarche exposée, ni celle des recherches menées, ni l'intérêt de la volonté d'implication, bien au contraire. Elles ne portent pas non plus sur le choix des outils. Je crois tout à fait nécessaire que de tels choix soient faits et il est vraisemblable que les signataires de cette charte aient fait, en fonction de leur expérience, le meilleur choix possible. Le problème vient, selon moi, du choix de l'exprimer sous la forme d'une «doctrine » et d'avoir donné à celle-ci la forme d'une "charte ». Ces termes sont très forts et constituent une revendication d'optimalité du choix réalisé. Or, je sais d'expérience que d'autres modélisateurs «impliqués » doivent faire - et font - d'autres choix et je formule l'hypothèse que, contrairement à cette sorte de fermeture de la démarche sur elle-même que laissent entendre les termes employés par les collègues qui nous proposent cette réflexion, les mises en commun des expériences pourraient être très positives.

Je crois que toutes les démarches de modélisation (la dite « bio-physico-mathématique »... et peut-être beaucoup d'autres) doivent être envisagées comme légitimes en tant qu'outils de la "modélisation d'accompagnement ». Qu'il risque d'y avoir des hypothèses implicites n'est pas grave, à condition de l'admettre et d'en tenir compte, par exemple, et, sans être certain d'y parvenir, en les rendant autant que faire se peut orthogonales aux questions explicites, comme cela a été théorisé dans la planification des expériences de l'époque fisherienne.

Sur un plan plus général, je ne crois pas qu'une recherche «impliquée » menée en interdisciplinarité puisse se définir à partir de la seule modélisation, même si cette dernière peut jouer un rôle déterminant dans sa conduite, comme cela apparaît très clairement et utilement dans le texte proposé. À ce titre, la distinction faite entre "production de connaissances » et « amélioration de la qualité des processus de décision » est effectivement très importante, mais il n'est pas sûr que la modélisation puisse jouer un rôle similaire sur chacun de ces deux axes. Si, par exemple, on considère que la validité d'un modèle (indépendamment du type de modèle considéré) peut être caractérisée par un ensemble de questions auxquelles il apporte des réponses jugées «bonnes », alors un modèle peut être le moyen d'obtenir et d'exprimer un résultat de recherche qui améliore effectivement la qualité des processus de décision. Des questions originales peuvent même alors s'imposer par la mise en œuvre de démarches de modélisation, et ces questions sont «de terrain » jusqu'à ce qu'au moins une autre discipline se les approprie. Cette appropriation entraîne un changement de statut de la question qui relève alors de la production de connaissances. Et, dans le même temps, la nécessité de la référence originale à la modélisation disparaît.

La réflexion dans laquelle prend place le texte proposé par le collectif ComMod peut ainsi utilement se poursuivre, sans nécessairement recourir à des termes utilisés dans le contexte de lois constitutionnelles. Cela peut se faire en précisant la nature et le statut épistémologique des questions de terrain et en examinant en quoi consiste, dans le contexte d'un collectif de recherche interdisciplinaire, l'implication de chacune des disciplines. Cela conduira forcément à poser la question de la relation entre les disciplines proprement dites de modélisation (mais peut-être s'agit-il de disciplines de représentation des connaissances et de l'observation, ou encore de l'information et de la communication) et les autres dans ce contexte de recherches interdisciplinaires impliquées. 